\title{
Polarization analysis of terahertz radiation generated by four-wave mixing in Air
}

\author{
Aurélien Houard, Yi Liu, Bernard Prade and André Mysyrowicz* \\ Laboratoire d'Optique Appliquée, ENSTA, Ecole Polytechnique, \\ CNRS UMR 7639, Palaiseau, 91761, France \\ *Corresponding author: andre.mysyrowicz@ensta.fr
}

\begin{abstract}
We examine the generation of terahertz by optical rectification of fundamental infrared beam with its first harmonic in ionized air. From polarization measurements we identify an important, yet so far unreported cross term $\left(\chi_{\text {xyxy }}^{(3)}+\chi_{\text {xyyx }}^{(3)}\right)$ of the nonlinear susceptibility tensor. Omission of this term leads to an error by a factor $10^{6}$ of the $\mathrm{THz}$ intensity obtained with certain polarization configurations.

OCIS codes: 260.3090 Infrared, far, 190.4380 Nonlinear optics, four-wave mixing, 350.5400 Plasma.
\end{abstract}

Discovered by Cook and Hochstrasser [1], the technique consisting in focusing a femtosecond pulse $(\omega)$ together with its first harmonic $(2 \omega)$ in air appears to be one of the most attractive methods to produce terahertz $(\mathrm{THz})$ radiation in the laboratory. It uses a widely available nonlinear medium (air at atmospheric pressure) as a source of $\mathrm{THz}$. In contrast to conventional $\mathrm{THz}$ emitters based on photo excitation of semiconductors or optical rectification in crystals, there is no upper limit in laser pulse intensity due to sample damage. THz pulse with broadband spectrum spanning over more than a decade, and peak field amplitude as high as $400 \mathrm{kV} / \mathrm{cm}$ has been reported with this method [2].

Two conditions appear to be necessary for a successful THz operation with pulses at $\omega$ and $2 \omega$. First, efficient $\mathrm{THz}$ generation requires the presence of plasma. This plasma can be produced when the femtosecond laser intensity is sufficiently high to ionise air molecules. It also requires a precise phase between the waves at $\omega$ and $2 \omega$. Two interpretations have been proposed to explain the THz generation. The first is a phenomenological $\chi^{(3)}$ approach. In this picture, mixing of the incident waves produces a down converted frequency at the THz frequency. The rectified term from the third order polarization is proportional to $\chi^{(3)} \mathbf{E}^{2 \omega}(t) \mathbf{E}^{\omega}(t) \mathbf{E}^{\omega}(\mathrm{t}) \cos \theta$ where $\theta$ is the phase difference between the waves at $\omega$ and $2 \omega$ [1]. Therefore, for optimum THz output, the phase $\theta$ should be normally 0 or $\pi$. The other interpretation is based on the production of a directed net current of electrons during the ionization process. An anisotropic current is produced in the tunnel ionization regime provided that the phase $\theta$ is $\pi / 2$ [3]. Recently, Thomson et al. have raised doubt on the validity of the $\chi^{(3)}$ approach [4]. They find important deviations from the $\chi^{(3)}$ model for the total THz signal. They interpret these deviations as being due to important higher order contributions $\left(\chi^{(5)}, \chi^{(7)}\right.$, etc...).

In this paper we re-examine carefully the polarization dependence of the $\mathrm{THz}$ signal. We show that the data can be reconciled with the $\chi^{(3)}$ model by properly taking into account a so far neglected term of the nonlinear susceptibility tensor. This term was omitted previously because it was assumed that the polarization of the laser is left unchanged during propagation in the BBO crystal used to generate the second harmonic wave.

It is possible to test the validity of the $\chi^{(3)}$ model by checking the polarization dependence of the $\mathrm{THz}$ signal. For instance, it is well known that in an isotropic medium such as air, the following relations should hold for the components of the third order susceptibility tensor $\chi^{(3)}(0,2 \omega, \omega, \omega)$ :

$$
\begin{aligned}
& \chi_{x y x y}^{(3)}=\chi_{y x y x}^{(3)}, \\
& \chi_{x y y x}^{(3)}=\chi_{y x x y}^{(3)}, \\
& \chi_{x x y y}^{(3)}=\chi_{y y x x}^{(3)},
\end{aligned}
$$




$$
\begin{gathered}
\chi_{x x x x}^{(3)}=\chi_{y y y y}^{(3)}=\chi_{x x y y}^{(3)}+\chi_{x y y x}^{(3)}+\chi_{x y x y}^{(3)}, \\
\chi_{x y y y}^{(3)}=\chi_{y x x x}^{(3)}=0 .
\end{gathered}
$$

Up to now, only partial measurement of the different tensor elements has been made, with conflicting results. Using set up (a) shown in Fig. 1, Kress et al. [5] have measured the dependence of the $x$ component (component parallel to the $\omega$ incident beam) of the $\mathrm{THz}$ signal as a function of angle $\alpha$ between the incident polarization vector at $\omega$ (set along $x$ ) and the extraordinary refractive index axis (or neutral line) of the BBO crystal which could be rotated. They explain their results by assuming that the predominant term of the $\chi^{(3)}$ tensor is the element $\chi^{(3)}{ }_{\mathrm{xxxx}}$, for which all polarization vectors at $\omega, 2 \omega$ and $\omega_{\mathrm{THz}}$ are aligned. According to their interpretation, no $y$-component of $\mathrm{THz}$ radiation is expected in this case. Bartel et al. [2] on the other hand find that the $\chi_{\text {xxyy }}^{(3)}$ term is predominant, a result incompatible with Kress. Xie et al. [6] repeated the experiment using separated beams at $\omega$ and $2 \omega$ (see Fig. 1(b)). With such a set up the polarization directions of the fields at $\omega$ and $2 \omega$ can be varied independently. Their results confirmed that $\chi_{\mathrm{xxxx}}^{(3)}$ is important.
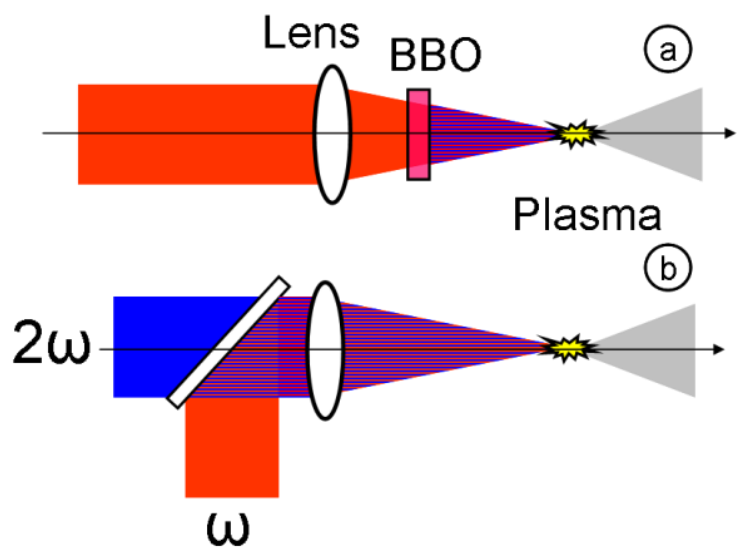

Fig. 1. Experimental setups. In setup (a), frequency $2 \omega$ is produced by a BBO crystal which can be rotated around the $z$ propagation axis. Because of the crystal birefringence, it is necessary in this case to consider the induced change of polarization at $\omega$. In setup (b), the polarization vectors at $\omega$ and $2 \omega$ can be varied independently.

However, with the detection techniques used, these authors could not measure the THz component orthogonal to the polarization vector of the field at $\omega$. We have repeated measurements of the dependence of the THz signal as a function of the polarization directions of the beams at $\omega$ and $2 \omega$ using both setups shown in Fig. 1. As a THz detector we use a heterodyne detector which records the frequency component around $0.1 \mathrm{THz}$. The advantage of this detector as compared to the more elaborate systems used by others is the fact that one can easily measure the THz components polarized along $x$ and $y$ independently of the laser polarization vectors. The laser pulse had a duration of $50 \mathrm{fs}$, a central frequency $\omega=800 \mathrm{~nm}$ and an energy below $300 \mu \mathrm{J}$. A lens with focal length $\mathrm{f}=25 \mathrm{~cm}$ was used to focus the laser. Results obtained with setup (a) are shown in Fig. 2. Also shown is a fit adopting the model from Kress et al. (blue dotted curve). As can be seen, the $x$ component of the THz is well fitted but the model fails by 6 orders of magnitude for the $y$ component. On the other hand, these results can be well fitted, as shown by the continuous red curves, provided the effect of birefringence of the BBO crystal on the $\omega$ beam is taken into account. The field components at $\omega$ after the crystal can be expressed as:

$$
\left\{\begin{array}{l}
E_{x}^{\omega}=E_{0} \sin \alpha \cos \alpha\left[\cos \left(\omega t-\varphi_{e}{ }^{\prime}\right)-\cos \left(\omega t-\varphi_{0}\right)\right], \\
E_{y}^{\omega}=E_{0}\left[\sin ^{2} \alpha \cos \left(\omega t-\varphi_{e}{ }^{\prime}\right)+\cos ^{2} \alpha \cos \left(\omega t-\varphi_{0}\right)\right],
\end{array}\right.
$$

and the field at $2 \omega$ as: 


$$
\left\{\begin{array}{l}
E_{x}^{2 \omega}=d_{e f f} \cos ^{2} \alpha \cos \alpha E_{0}{ }^{2} \cos \left(2 \omega t-2 \varphi_{0}\right), \\
E_{y}^{2 \omega}=d_{e f f} \cos ^{2} \alpha \sin \alpha E_{0}{ }^{2} \cos \left(2 \omega t-2 \varphi_{0}\right),
\end{array}\right.
$$

where $d_{\text {eff }}$ is the effective conversion coefficient of the crystal from $\omega$ to $2 \omega$, and $\varphi_{0}$ and $\varphi_{e}$ are the phase delays of the ordinary and extraordinary lights respectively. Taking into account the two components $E_{x}$ and $E_{y}$ at $\omega$, a general expression for the $\mathrm{THz}$ field is given by:

$$
\left\{\begin{array}{l}
E_{x}{ }^{T H z}=\chi_{x x x x}^{(3)} E_{x}^{2 \omega} E_{x}^{\omega} E_{x}^{\omega}+\left[\chi_{x y x y}^{(3)}+\chi_{x y y x}^{(3)}\right] E_{y}^{2 \omega} E_{x}^{\omega} E_{y}^{\omega}+\chi_{x x y y}^{(3)} E_{x}^{2 \omega} E_{y}^{\omega} E_{y}^{\omega}, \\
E_{y}{ }^{T H z}=\chi_{y y y y}^{(3)} E_{y}^{2 \omega} E_{y}^{\omega} E_{y}^{\omega}+\left[\chi_{y x y x}^{(3)}+\chi_{y x x y}^{(3)}\right] E_{x}^{2 \omega} E_{y}^{\omega} E_{x}^{\omega}+\chi_{y y x x}^{(3)} E_{y}^{2 \omega} E_{x}^{\omega} E_{x}^{\omega} .
\end{array}\right.
$$

Using these expressions, a best fit can be obtained if one assumes a tensor element $\left(\chi_{\mathrm{xyxy}}^{(3)}+\chi_{\mathrm{xyyx}}^{(3)}\right)$ contributing with same magnitude as term $\chi^{(3)}{ }_{\mathrm{xxx}}$ (see red curve in Fig. 2).

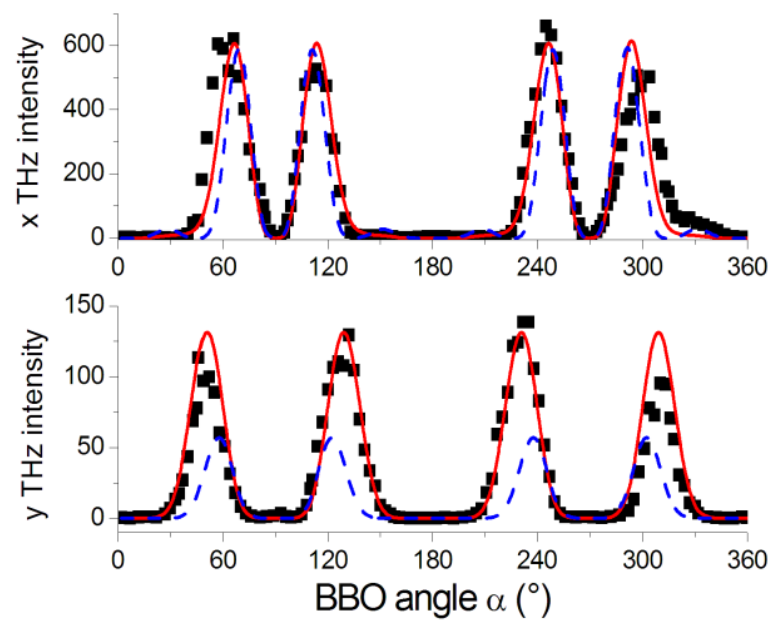

Fig. 2. Variation of the THz intensity (in arbitrary units) with the angle $\alpha$ of the BBO crystal (black dots). Comparison with two fits considering $\chi_{\text {xxx }}^{(3)}$ only (blue dashed line) and considering $\chi_{\text {xxx }}^{(3)}$ and $\chi_{\text {xyxy }}^{(3)}+\chi_{\text {xyxy }}^{(3)}$ (red continuous line). Note that in the $y$ plot, the blue curve has been multiplied by $10^{6}$.

In order to confirm the importance of the term $\chi^{(3)}{ }_{\text {xуху }}$ deduced from this analysis, we have used setup (b) (shown in Fig. 1), where the polarizations and intensities of the fields at $\omega$ and $2 \omega$ can be varied independently. To obtain components of the field at $\omega$ along $x$ and $y$ at the same time we used a $\omega$ field polarized at $45^{\circ}$ with respect to the field at $2 \omega$. In this way, all $\chi^{(3)}$ components can be measured. For each measurement the THz signal was optimized by adjusting the time delay between $\omega$ and $2 \omega$. As seen in Fig. 3 , there are two thresholds for the appearance of different terms of the $\chi^{(3)}$ tensor. The first threshold at 25 $\mu \mathrm{J}$ can be identified with the onset of ionization. This is confirmed by a measurement of the onset of current circulating between two charged electrodes placed in the focal region of the laser. It confirms the essential role of the plasma in the production of $\mathrm{THz}$, as recognized earlier $[5,6]$. The second threshold at $125 \mu \mathrm{J}$ is tied to the appearance of term $\chi_{\text {xyyy }}^{(3)}$ which becomes comparable in strength to all other contributions. We postpone the discussion of this term and concentrate on the situation for laser pulse energy at $\omega$ comprised between $25 \mu \mathrm{J}$ and $125 \mu \mathrm{J}$. Table I shows the different tensor elements extracted from this study. These results are in good overall agreement with the $\chi^{(3)}$ model assuming local isotropy. We note first that the THz field amplitude varies linearly with the $\omega$ laser pulse energy, in agreement with the $\chi^{(3)}$ model. Furthermore, the only important term measured besides $\chi_{\text {xxx }}^{(3)}$ is the term $\left(\chi_{\text {xyxy }}^{(3)} \chi_{\text {xyyx }}^{(3)}\right)$ which must therefore be equal to $\chi^{(3)}{ }_{\mathrm{xxxx}}$, according to the third row of equation (1). This is indeed the case, except for a minor contribution from term $\chi_{\text {yуху }}^{(3)}$ which implies local anisotropy. A small deviation for strict local isotropy has been also reported earlier by Xie et al. [6]. 


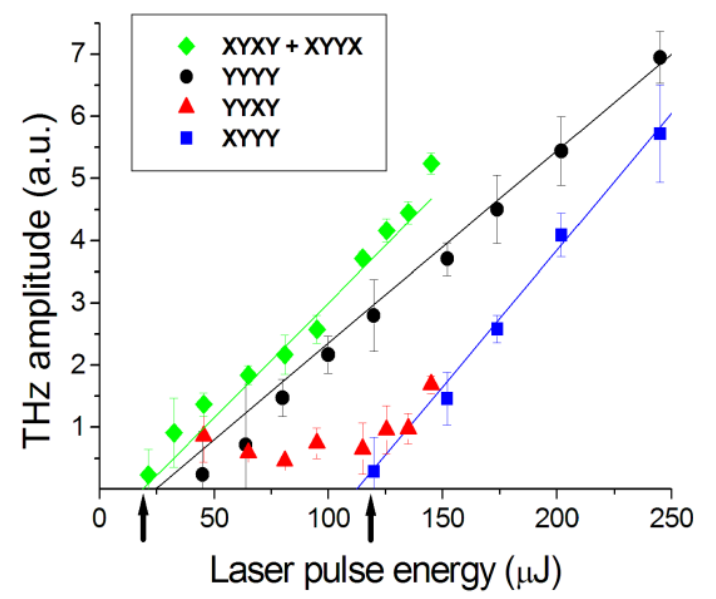

Fig. 3. Evolution of the THz field as function of the laser pulse energy, for four different components of $\chi^{(3)}$. The two arrows indicate the thresholds for the appearance of terms.

Finally, examining the situation above $125 \mu \mathrm{J}$ in Fig. 3, we note the appearance of term $\chi^{(3)}$ xyyy which becomes comparable to the other contributions at higher laser energies. In fact, the $\mathrm{THz}$ radiation from such a term, for which the THz polarization is orthogonal to all other laser fields, corresponds to a radially polarized wave. We checked that the radiated waves presents a two-lobes pattern characteristics of the conical wave emitted by plasma filaments, as already reported with a single laser frequency [7,8]. In the present case, filament formation, which breaks spatial isotropy, is formed by the combination of the fields at $\omega$ and $2 \omega$. A more detailed study of this high intensity regime is in preparation.

In conclusion, a careful study of the polarization properties of the THz radiation produced by mixing of $\omega$ with $2 \omega$ in the presence of plasma, shows that this emission can be explained in a large intensity interval by a $\chi^{(3)}$ process in a quasi-isotropic medium. This is in contradiction with recent claims [4]. However, important deviations from spatial isotropy are observed at higher laser intensities.

Table 1. Comparison between the different $\chi^{(3)}$ coefficients for laser energies below $100 \mu \mathrm{J}$. The table presents the results obtained by Xie et al. [6] and our results. All measurements are normalized to $\chi_{\text {xхxх }}^{(3)}$.

\begin{tabular}{|c|c|c|}
\hline $\begin{array}{c}\mathrm{THz}, \\
2 \omega, \omega, \\
\omega \\
\end{array}$ & $\begin{array}{l}\text { Normalized } \\
\text { THz field (Xie } \\
\text { et al.) }\end{array}$ & $\begin{array}{l}\text { Normalized THz } \\
\text { field (our } \\
\text { measurements) }\end{array}$ \\
\hline$x x x x$ & 1 & 1 \\
\hline$y y x x$ & 0.05 & 0.1 \\
\hline$x y x x$ & 0.04 & 0.2 \\
\hline xyyy & 0.1 & 0.3 \\
\hline yyxy & - & 0.15 \\
\hline $\begin{array}{c}x y x y+ \\
\text { xyyx }\end{array}$ & - & 0.98 \\
\hline
\end{tabular}

\section{References}

1. D.J. Cook and R.M. Hochstrasser, "Intense terahertz pulses by four-wave rectification in air," Opt. Lett. 25, 1210 (2000).

2. T. Bartel, P. Gaal, K. Reimann, M. Woerner and T. Elsaesser, "Generation of single-cycle THz transients with high electric-field amplitudes," Opt. Lett. 30, 2805 (2005).

3. K.Y. Kim, J.H. Glownia, A.J. Taylor and G. Rodriguez, "Terahertz emission from ultrafast ionizing air in symmetry-broken laser fields", Opt. Express 15, 4577 (2007). 
4. M.D. Thomson, M. Kreß, T. Löffler and H.G. Roskos, "Broadband THz emission from gas plasma induced by femtosecond optical pulses: From fundamentals to applications", Laser Photonics Rev. 1, 349 (2007).

5. M. Kress, T. Löffler, S. Eden, M. Thomson and H.G. Roskos, "Terahertz-pulse generation by photoionization of air with laser pulses composed of both fundamental and second-harmonic waves", Opt. Lett. 29, 1120 (2004).

6. X. Xie, J. Dai and X.-C. Zhang, "Coherent Control of THz Wave Generation in Ambient Air", Phys. Rev. Lett. 96, 075005 (2006).

7. C. D'Amico, A. Houard, M. Franco, B. Prade, A. Couairon, V.T. Tikhonchuk, A. Mysyrowicz, "Conical Forward THz Emission from Femtosecond-Laser-Beam Filamentation in Air," Phys. Rev. Lett. 98, 235002 (2007).

8. C. D'Amico, A. Houard, S. Akturk, Y. Liu, J. Le Bloas, M. Franco, B. Prade, A. Couairon, V.T. Tikhonchuk, and A. Mysyrowicz, "Forward THz radiation emission by femtosecond filamentation in gases: theory and experiment," New Journal of Physics 10, 013015 (2008). 